\title{
A qualitative investigation into the follow-up support offered to patients after an NHS obesity weight management programme in Liverpool
}

\author{
R. J. Webb ${ }^{1}$, I. G. Davies ${ }^{1}$, B. Johnson ${ }^{2}$ and J. C. Abayomi ${ }^{1}$ \\ ${ }^{1}$ Faculty of Education, Community and Leisure, Liverpool John Moores University, Liverpool, UK and ${ }^{2}$ Abercromby Family \\ Centre, Grove Street, Liverpool, UK
}

The prevalence of obesity has been increasing in Liverpool over the last 20 years ${ }^{(1)}$. In response a weight management programme was devised by Liverpool Community Health using existing related services. The service involves dietitians working towards facilitating dietary and lifestyle changes in local obese NHS patients via a 12 week education programme. Follow-up support is highlighted in the literature as a required element for success in weight management, however there is a lack of existing qualitative research focussing on this area ${ }^{(2)}$. This study investigated patients' lived experiences of the programme; specifically regarding follow-up support. Participants $(n=16)$ were recruited from the programme to engage in a semi-structured focus group after their final education session with an emphasis on post-programme support. Data was audio-recorded, transcribed verbatim and analysed using constant comparison analysis. A coding frame was developed until saturation was reached. The table outlines themes which emerged from the data and future recommendations.

\begin{tabular}{ll}
\hline Themes involving follow up support & \multicolumn{1}{c}{ Recommendations } \\
\hline Lack of follow-up support from LWMP & Online support programme \\
Lack of support from family and friends & Follow-up phone calls from nurse \\
Over-dependence on healthcare professionals & Weight management support inventory \\
& Sessions run by NHS Health Trainers \\
\hline
\end{tabular}

Regardless of many positive comments, participants frequently complained about a lack of follow-up support from the programme and from family and friends. Some explained how they were often insulted and belittled by those closest to them and were vehemently against involving these people in their weight management. Follow-up weigh-in groups based around peer support were suggested, however these were also met with criticism as participants mentioned they would be unhappy to engage unless sessions were run by a dietitian or qualified health professional. Despite favourable remarks concerning the social elements of the programme and the acquisition of new skills and knowledge, many participants still felt unable to continue without suitable follow-up support and excessive practitioner dependence, therefore deflecting accountability for their health away from themselves. The results show that for changes made during programme to be sustainable, patients need a robust, post-programme support network where they become responsible for their own actions and are encouraged to set and work towards their own goals. Proposed solutions to this are the introduction of online support programmes, follow-up dietitian phone calls and weight management support inventories to assess the level of social support needed ${ }^{(3)}$. Furthermore, NHS Community Food Workers have also been effective in offering individualised support for behaviour changes and may too be useful for assisting with follow-up sessions ${ }^{(4)}$.

1. Healthy Weight: Healthy Liverpool (2008) Available at: www.liverpoolpct.nhs.uk (Accessed 18/02/13).

2. Perry K, Hickson M \& Thomas J (2011) Journal of Human Nutrition and Dietetics 24, 301-302.

3. Reider S, \& Ruderman A (2009) Eating Behaviours 8, 39-47.

4. Cowbrough K (2008) Journal of Human Nutrition \& Dietetics 21, 193-195. 